\title{
Iron Status of Malarial Infected Pregnant Women: A Review
}

\author{
Obeagu Emmanuel Ifeanyi \\ Department of Medical Laboratory Science, Imo State University, Owerri, Nigeria. \\ *Corresponding Author: Obeagu Emmanuel Ifeanyi, Department of Medical Laboratory Science, \\ Imo State University, Owerri, Nigeria.
}

\begin{abstract}
Malaria and Iron deficiency are the predominant causes of severe anaemia in sub-Saharan Africa and have their respective deleterious impact on women of reproductive age, the neonates, infants and children. Iron is vital for the survival of the malaria parasite and the parasite is unable to thrive in iron-deficient environment. Different search databases were utilized in this review such as Pubmed, Google Scholar, Research gate, Academia Edu and so many others. The review showed that iron deficiency suppresses erythropoiesis, thereby reducing the opportunity for parasite to infect the host. Iron supplementation may be safe during pregnancy in malaria endemic areas.
\end{abstract}

Keywords: Iron status, pregnant women, Malaria

\section{INTRODUCTION}

Malaria is one of the world's deadliest and most geographically widespread human infectious diseases, causing hundreds of thousands of deaths per year (Natasha et al., 2017). Malaria infections contribute significantly to the worldwide burden of anemia, and measures taken to decrease malaria at the population level frequently decrease anemia prevalence (Natasha et al., 2017). Malaria and Iron deficiency are the predominant causes of severe anaemia in subSaharan Africa and have their respective deleterious impact on women of reproductive age, the neonates, infants and children. The vulnerability of children and pregnant women to malaria is because children have naive immune system while the immune system of pregnant women is potentially compromised. Consequently, the two groups are considered to be the highest risk population for malaria-related death. Iron is absorbed in the gastrointestinal tract and is essential for erythropoiesis (Okamgba et al., 2017). In most cases, the amount of iron absorbed is not always enough for sustenance of pregnancy and may result to iron deficiency. The major reason for iron deficiency during pregnancy is that pregnancy places tremendous increase in the body's need for iron in order to match with the increase in plasma volume of the pregnant woman and the fetal need for proper development (Bothwell T. H, 2000). The need culminates in depleted iron stores. The progressive depletion of iron-stores will eventually result to Iron Deficiency Anaemia (IDA) (Dreyfuss et al., 2000). Body iron status is usually assessed by considering haemoglobin, red cell indices and serum Ferritin concentrations along with evidence of inflammation, infection and liver disease (Worwood et al., 2012).

More than $38 \%$ of pregnant women worldwide are anemic, with the prevalence greatest in subSaharan Africa and parts of Asia (WHO, 2015). In Africa and Asia, anemia directly or indirectly contributes to one-quarter of maternal deaths (WHO, 2015). Consequences of antenatal anemia include premature delivery and low birth weight. Universal iron supplementation is recommended by the WHO for all pregnant women in a setting where anemia prevalence in this population exceeds 40\% (WHO, 2015). However, only half of anemia cases in pregnancy worldwide (including only $44 \%$ of cases in Africa and $47 \%$ of cases in Asia) are attributed to iron deficiency and amenable to iron supplementation (WHO, 2015). The use of iron to treat other causes of anemia (including malaria, inflammation, and hemoglobinopathy) may not be beneficial, may represent a missed opportunity to identify and treat an alternative condition, and in some cases, may be actively harmful because it could exacerbate the risk of infection or iron overload (Jones et al., 2015). However, such cases would likely be misdiagnosed and iron supplementation provided if a decision for iron intervention was based on a diagnosis of anemia, or if universal 
iron interventions are provided. Therefore, an appropriate case definition of iron deficiency in pregnancy could be of value in correctly identifying women who would most benefit from iron interventions (Jones et al., 2015).

Pregnancy is a period in the life cycle of a woman when additional nutrients are needed to meet the metabolic and psychological demands as well as the increased requirements of the growing fetus and placenta. Many anatomic, biochemical and physiologic changes occur during pregnancy to maintain a healthy environment for the growing fetus (Akinbo et al., 2019). Maternal nutrition does not only impact the mother in terms of morbidity but also on the growth and development of the fetus. The quality of nutrition in the first 1000 days beginning from the mother's pregnancy through her child's second birthday is a critical window when a child's brain and body develop rapidly and good nutrition is essential to lay the foundation for a healthy and productive future (Akinbo et al., 2019). Undernutrition is an underlying cause of death and it leaves millions more with lifelong physical and mental impairments (Paul et al., 2011). Poor quality diet due to inadequate intake of animal source foods particularly in developing countries has been implicated as the major cause of micronutrient deficiencies. In addition, women who avoid meat and/or milk in developed countries have been reported to have higher risk of micronutrient depletion during pregnancy and lactation (Akinbo et al., 2019). Several micronutrient deficiencies have been established to contribute to abnormal prenatal development and/or pregnancy outcome. The common micronutrients are vitamins $\mathrm{A}, \mathrm{B}, \mathrm{C}, \mathrm{D}$ and $\mathrm{E}$, calcium, zinc, copper, iron, and magnesium (Akinbo et al., 2019).

Iron is essential for many metabolic processes, including oxygen transport and regulation of cell growth and differentiation (Mary et al., 2014). Iron deficiency most prominently causes anaemia, limiting oxygen delivery to cells, but iron deficiency can also cause dysfunction of the epithelia, muscle, and the nervous system. On the other hand, excess amounts of iron in cells and tissues can result in tissue toxicity. Iron requirements increase nearly 10 -fold during pregnancy from $0.8 \mathrm{mg} /$ day in the first trimester to $7.5 \mathrm{mg} / \mathrm{day}$ in the third trimester. During pregnancy, iron is needed to support placental and fetal growth sustain the increase in maternal red blood cell mass, and compensate for blood losses that will occur during delivery (Mary et al., 2014). The Recommended Dietary Allowance (RDA) for iron increases from 18 $\mathrm{mg}$ /day for non-pregnant females to $27 \mathrm{mg}$ /day during pregnancy (Mary et al., 2014). Importantly, the RDA does not take into account varying bioavailability of iron among individuals. The median dietary iron intake among pregnant women is approximately $15 \mathrm{mg} /$ day, an amount insufficient to support the increased demand during pregnancy. Therefore, many major health organizations recommend iron supplementation throughout pregnancy. The Centers for Disease Control and Prevention recommends routine low-dose iron supplementation (30 mg/day) for all pregnant women and the Institute of Medicine also supports iron supplementation during pregnancy (WHO, 2015).

Iron is important for early placental development, which maintains pregnancy and provides nutrients and oxygen to the developing fetus. Iron deficiency can adversely impact birth outcomes and result in preterm birth and low birth weight (Mary et al., 2014). The mechanisms by which iron deficiency may affect birth outcomes are unknown, but the effects of hypoxia, oxidative stress, and increased risk of infection have been proposed as potential pathways (Mary et al., 2014). Hypoxia, as a result of iron deficiency, could initiate a stress response, including the release of corticotropin-releasing hormone from the placenta and increased production of cortisol by the fetus, both of which are associated with increased risk of preterm birth. Iron deficiency is associated with increased oxidative stress and this could damage the placenta during early development. Iron deficiency can also negatively impact the immune response and thus increase the risk of infection during pregnancy (Mary et al., 2014). (Namama, 2015) agreed that serum iron and Total Iron Binding Capacity (TIBC) are frequently abnormal in pregnancy and so they are of little help in diagnosis of iron deficiency anemia during pregnancy. Serum ferritin was found to be low in most mothers; ferritin level is the best parameter for assessment of iron and is unreliable during pregnancy (Namama, 2015). During pregnancy, haemoglobin levels in woman are naturally lower than when she is not pregnant. This is because the fluid (plasma) increases by about $50 \%$ during pregnancy (peaking at about 32 weeks) (Namama, 2015) and the increased plasma dilutes the red cells, making their level drop while pregnant women with malaria infection and with or without anemia have 
relatively low ferritin concentrations, low plasma iron concentrations, and low transferrin saturation as well as high TIBC concentrations, suggesting some degree of iron deficiency (Susanneet al., 2011).

The interactions between malaria and iron have only lately begun to be understood at the molecular level. Primarily, the discovery of the iron regulatory hormone hepcidin has given us new understanding of human iron physiology and pathophysiology. Hepcidin serves to block iron absorption from the diet and also to route iron in the body into macrophages and away from the serum. Hepcidin plays a complex but vital role in both the iron restriction that occurs during malaria infection, and in determining iron status and thereby influencing disease susceptibility.

\section{IRON METABOLISM}

Systemic mammalian iron metabolism is controlled at the level of iron absorbance from the diet and iron recycling through macrophages. Approximately $1 \mathrm{mg}$ of iron is absorbed from the diet every day, roughly equivalent to the iron that is lost daily in poorly regulated activities such as sweating, any bleeding, and the sloughing off of enterocytes. At the same time, iron already in the body is constantly being recycled as macrophages phagocytose senescent or damaged red blood cells, digest the haeme and extract the iron they contain, and export that iron back into the circulation. The export of iron across the basolateral membrane of enterocytes and the recycling of iron through macrophages are dependent on the same transport protein: ferroportin, the sole currently identified mammalian iron export protein (Natasha et al., 2017).

The release of iron from enterocytes into the bloodstream is the final step of absorption of iron from the diet. Hepcidin, discovered a decade ago by three groups working independently (Natasha et al., 2017), is a 25amino acid protein that binds to ferroportin and causes it to be internalized and degraded. The effect of ferroportin inhibition by hepcidin is therefore to block uptake of dietary iron from the intestine, and to increase the accumulation of iron in macrophages. The result is a decrease in serum iron levels, which routes iron away from pathogens that could potentially exploit circulating iron, but may also render the host anaemic by restricting iron availability to the erythron. Evidence from both animal models and from human genetic lesions suggests that the hepcidin-ferroportin interaction is functionally non-redundant (Natasha et al., 2017).

\section{IRON IN THE FOOD}

Iron is the most abundant element on earth, with potential of high toxicity to living cells. However, it has poor bioavailability, and efforts have been made to provide iron for everybody, notably by food fortification within rice (Sophie et al., 2014), because it represents one of the most essential nutriments for human beings. Rice and most staple cereals contain low iron levels, since most iron-containing components are lost during grain processing. Populations with monotonous diets consisting mainly of cereals are especially prone to iron deficiency, which affects about two billion people.

Food fortification programs to supplement nutrition with iron have not been very successful. One alternative solution is iron biofortification. Different approaches have been studied, including conventional breeding and directed genetic modification, which offer the most rapid way to develop iron-rich rice plants (Sophie et al., 2014). Biofortification of crops is also an interesting approach, and at least two complementary approaches have been successfully adopted to increase the concentrations of bioavailable mineral elements in food crops (White et al., 2009). First, application of mineral fertilizers and/or improving the solubilization and mobilization of mineral elements in the soil has been implemented. Secondly, crops have been developed with increased abilities to acquire mineral elements and accumulate them in edible tissues. In the same context, it seems necessary to highlight the efforts made by some investigators, who developed high-iron rice, using transgenic approaches. They created highiron rice by insertion of soybean ferritin gene under the control of the endosperm-specific glutelin promoter into the genome of the Indica rice line populations (Gayen et al., 2013). This is why the knowledge of the iron content of various ailments as well as of the factors influencing its absorption should be improved (Gayen et al., 2013)

\section{IRON IN THE BODY}

Males contain about 4,000 mg of iron, of which $2,500 \mathrm{mg}$ are within erythrocytes; $1,000 \mathrm{mg}$ is stored in spleenic and hepatic macrophages, and the rest is distributed in various proteins such as Myoglobulin (Mb), cytochromes, or other 
ferroproteins. Only about $3 \mathrm{mg}$ are bound to plasma Transferrin and constitute the mobile iron compartment which supplies the various intracellular iron stores. About $1-2 \mathrm{mg}$ of iron is lost every day, through skin and enteric desquamation and minor blood losses. This loss is balanced by intestinal absorption. Therefore, iron recycling accounts for most of the iron homeostasis in human. The situation is different in menstruating women where there are controversial discussions about iron stores, ferritin, and Haemoglobin levels (Sophie et al., 2014). It appears that lower Haemoglobin and ferritin values in menstruating women have been accepted as normal rather than possibly representing widespread iron deficiency. The situation is even more complex in pregnant women; nevertheless, iron substitution has been shown to be beneficial for them (Casgrain et al., 2012). Similarly, increased iron demand occurs during infancy and childhood due to growth and development demands.

\section{INTESTINAL IRON ABSORPTION}

Iron absorption is the result of complex mechanisms that takes place in the upper parts of the gut, notably in the duodenum and the proximal jejunum (Fuqua et al., 2012). On the brush border of enterocytes, various iron import proteins are present, and specific pathways of absorption have been described for the two ionic forms of iron ( $\mathrm{Fe} 2+$ and $\mathrm{Fe} 3+$; both being nonhaeme iron molecules) and also for iron associated with haeme (haeme iron) (Fuqua et al., 2012). Non-haeme iron is associated with various storage proteins, including ferritin, whereas haeminic iron is present within haemoproteins such as Myoglobulin ( $\mathrm{Mb}$ ) or Haemoglobin $(\mathrm{Hb})$. At acidic $\mathrm{pH}$ in the stomach, haeme is dissociated from haemoproteins, whereas non-haeme iron stabilizes in its reduced form $(\mathrm{Fe} 2+)$. It is important to note that nonhaeme iron is captured by several complexes which can interfere with its absorption, notably plant-derived phytates or tannins (Yersin et al., 2012). Ascorbic acid and other acidic components derived from the diet can increase iron absorption. Nevertheless, it is known that different pathways exist for the absorption of non-haeme iron and haeme iron. The distinction is of potential interest, because it has been shown that high haeme iron intake leads to increased body iron stores which are significantly associated with higher risk to develop type 2 diabetes mellitus (Bao et al., 2012). In contrast, total dietary iron, non-haeme iron, and intake of iron supplements were not associated with type 2 diabetes mellitus. Several well regulated gate keeper proteins are expressed in the duodenum enterocytes and are differently regulated as compared to the same proteins in liver cells. DMT1 is the most important transporter of ferrous iron $(\mathrm{Fe} 2+)$, Of note, ferric reductase activities due to duodenal cytochrome B and STEAPs (Six Transmembrane Epithelial Antigen of the Prostate Proteins) are present on the brush border of duodenum allowing reduction of ferric to ferrous iron, thus facilitating its absorption by DMT1. (Choi et al., 2012).

Haeme iron is an important nutritional source of iron in carnivores and omnivores that is more readily absorbed than non-haeme iron derived from vegetables and grain. Most haeme is absorbed in the proximal intestine, with absorptive capacity decreasing distally, and the role of specific proteins such as hephaestin has been deciphered (Choi et al., 2012). Haeme Carrier Protein1, which presents homology to bacterial metal-tetracycline transporters, mediates haeme uptake by the cells at the luminal brush border membrane of duodenal enterocytes. Haeme Carrier Protein1 (HCP1) and messenger Ribonucleic acid (mRNA) has been shown to be highly expressed in the duodenum and regulated by hypoxia and by Iron Regulatory Protein(IRPs).

\section{IRON AND ERYTHROPOIESIS}

Erythroid precursors need much more iron than any other type of cells in the body, and, as previously mentioned, they take up iron almost exclusively through plasma transferrrin1. Iron transport into mitochondria is provided by mitoferrin-1, the mitochondrial iron transporter 1 of erythroid precurors (Choi et al., 2012). Mitoferrin-1 interacts with an ATP-binding transporter and binds to ferrochelatase to form an oligomeric complex allowing iron uptake and haeme biosynthesis (Chen et al., 2010).

Erythroid cells contain adaptative mechanisms to face iron deficiency and a class of kinases activated by different cellular stresses. For example, during iron deficiency, and as haeme concentration drop, haeme dissociate from the haeme-regulated inhibitor kinase known as Heptad Repeat1 (HRI), leading to its autophosphorylation and phosphorylation of the $\alpha$-subunit of eukaryotic translation initiation factor 2 (Suragani et al., 2012). Heptad RepeatIdeficient mice have allowed identifying HRI as a protector of apoptosis and being involved in the formation of microcytes. 


\section{IRON TRANSPORTATION IN BLOOD}

Transferrin is the main protein involved in iron transport in plasma. Normally, between 20 and $40 \%$ of the binding sites of the protein are occupied by ferric iron. The diagnostic value of Transferrin (Tf) has just been reviewed. It proved to be a useful parameter for assessing both iron deficiency and iron overload. The saturation of $\mathrm{Tf}$ is a strong indicator of iron overload. However, from a physiological point of view, the iron binding capacity of plasma Tf is often exhausted, with concomitant generation of non-Tf-bound iron (NTBI) as observed in transfused patients. Using fluorescent tracing of labile iron in endosomal vesicles and cytosol, (Sophie et al., 2014) showed that NTBI fractions derived from sera of polytransfused thalassemia major patients entered cells via endocytosis. Erythrocyte precursors restrictively take up iron by using Tfr, notably Tfr1, whereas hepatocytes and other non-erythroid cells are also able to use NTBI. Iron-Tf binds to Tfr, and the complexes are internalized within the cell by the endosomal recycling vesicles. Thus, the $\mathrm{Tf}$ cycle is dependent on the Tf-Tfr complex trafficking, involving internalization of the complex within endosome, followed by iron release upon acidification of the endosome and recycling of the Tf-Tfr complex to the cell surface. Each of these steps is mediated by a specific pathway and specific machinery (Sophie et al., 2014). Finally, at the cell surface, at neutral $\mathrm{pH}$, Tf dissociates from Tfr, and is used to repeat the iron cycle. In addition, Tfr is cleaved and shed as a soluble form (sTfr) into the extracellular and intravascular space. This shedding of Tfr1 is known for more than 30 years, and its assessment is well accepted as a diagnostic marker of iron-depleted erythropoiesis (Sophie et al., 2014). Very recently, the cleavage site as well as the cleaving proteases of membrane Tfr1 has been identified.

\section{MECHANISM OF IRON TRANSFER DURING PREGNANCY}

Iron $(\mathrm{Fe})$ is carried in the maternal blood bound to transferrin (Tf). The syncytiotrophoblast, the epithelium on placental villi that interfaces with maternal blood and participates in nutrient exchange, contains $\mathrm{Tf}$ receptors (TfR) on the surface facing maternal circulation. The binding of Fe-Tf to TfR depends on the $\mathrm{pH}$ levels, having a greater affinity at $\mathrm{pH} 7.4$ (Marry et al., 2014). After binding, the Fe-Tf/TfR complex is endocytosed, and the vesicle is acidified through activation of hydrogen pumps. The lower $\mathrm{pH}$ allows the dissociation of iron from the maternal Tf. Once released, iron is actively transported out of the vesicle into the cytosol where it is used for cellular processes, stored in ferritin or exported into fetal circulation. The TfR and Tf then return to the maternal surface of syncytiotrophoblast where $\mathrm{Tf}$ is released into the maternal circulation and the cycle repeats (Sophie et al., 2014).

Iron is exported from the basolateral side of the synctiotrophoblast into fetal circulation by ferroportin (Fpn), the only know iron exporter in vertebrates. In fact, knockout of Fpn in mice causes embryonic lethality due to severe iron deficiency of the embryo (Marry et al., 2014). Following iron export through Fpn, a ferroxidase (possibly zyklopen) oxidizes iron so it can be loaded onto fetal transferrin. In the last decade, there has been a revolution in our understanding of systemic iron homeostasis. Hepcidin is a peptide hormone that functions as both the homeostatic regulator of systemic iron metabolism and a mediator of host defense. Sensing of circulating iron and iron stores is thought to occur in the liver, which is the primary site of hepcidin production and secretion (Choi et al., 2012).

Hepcidin production can be assessed by measuring liver hepcidin mRNA levels (in animal models) or by measuring hepcidin peptide in the serum or plasma (in humans and mice) (Mary et al., 2014). Circulating hepcidin is rapidly excreted through the kidneys (half-life in circulation is several minutes), and the peptide can also be measured in urine. Because hepcidin production is predominantly regulated at the transcriptional level, hepcidin mRNA and protein levels show high correlation. Hepcidin controls the efflux of iron into plasma by regulating Fpn (Mary et al., 2014).

In addition to the placenta, ferroportin (Fpn) is located on tissues that actively export iron including intestinal enterocytes, reticulo endothelial macrophages, and hepatocytes. Hepcidin triggers Fpn degradation, reducing iron flux from these tissues thereby decreasing plasma iron concentrations and systemic iron bioavailability. Hepcidin production by the liver is simultaneously regulated by circulating and stored iron, erythropoietin activity, and inflammation. Therefore, at any time, hepcidin expression is determined by the interplay of these pathways and the relative strength of each of the individual signals (Marry et al., 2014). When body iron levels are elevated or 
inflammation or infection is present, liver hepcidin production is increased resulting in diminished Fpn expression. Conversely, when body iron levels are depleted or anemia or hypoxia exists, hepcidin expression is reduced, allowing for increased dietary iron absorption and mobilization from body stores via active Fpn. Furthermore, hepcidin concentrations markedly influence iron absorption and can affect the efficacy of iron repletion via supplemental or dietary sources. Therefore, given that iron accessibility is critical throughout pregnancy for both the mother and developing fetus, and hepcidin regulates systemic iron bioavailability, it is important to evaluate hepcidin concentrations in both uncomplicated and complicated pregnancies and throughout gestation (Sophie et al., 2014).

\section{EFFeCt OF Malaria on Iron Metabolism}

Erythropoietic iron requirement is met largely through recycling of senescent red blood cells through reticuloendothelial macrophages, where iron is repackaged onto transferrin for transport to the bone marrow (Andrew et al., 2010). Red blood cells are predominantly engulfed directly by reticuloendothelial macrophages; however, up to $10 \%$ of normal recycling occurs as a result of intravascular hemolysis. Hemoglobin is released and rapidly bound to haptoglobin, and the resultant complex is cleared by liver parenchymal cells or the CD163 macrophagal receptor (Andrew et al., 2010). Malaria-induced destruction of infected and noninfected red blood cells both stresses and impedes the capacity of reticuloendothelial macrophages to recycle iron back to the bone marrow. Intravascular rupture of parasitized red cells, macrophagal phagocytosis of both parasitized and unparasitized red cells, and hypersplenism all contribute to the pathophysiology of the anemia associated particularly with acute malaria. The local epidemiology and transmission characteristics of Plasmodium falciparum, together with local drug treatment and intermittent preventive therapy (IPT) practices, influence population immunity and resultant malarial clinical presentation as well as the epidemiology of anemia in a particular setting. These factors may also influence the disturbance of iron metabolism and erythropoietic response to iron supplementation. Acute malaria is associated predominantly with a picture of fever, hemolysis, and oxidant stress, whereas dyserythropoiesis predominates in the anemia of chronic malaria in a semi immune patient (Andrew et al., 2010). Acute malarial haemolysis represents a major stress on the homeostatic mechanisms of iron flux regulation that have evolved primarily to maintain erythroblast supply while minimizing the oxidant stress-associated effects of iron (Sophie et al., 2014).

\section{PATHOPHySIOLOGY OF MALARIA}

Malaria parasite is transmitted by the bite of a sporozoite-bearing female anopheline mosquito. Life cycle of malaria parasite is illustrated in Figure 3 below. Following the mosquito bite, initially, there is a period of pre-erythrocytic phase in the liver where they invade hepatocyte. Following this, the erythrocytic stage of infection occurs, which causes disease manifestations. During this stage, invasion of the erythrocyte by the parasite results in the consumption of haemoglobin and alteration of the red cell membrane. The sporozoite cells divide many times and, due to this, mature tissue schizonts develop (Richa et al., 2017). Each of the hepatocyte now contains many thousands of merozoites. This pre-erythrocytic stage is asymptomatic. After this exoerythrocytic stage, the liver schizonts rupture after few days, which now release many thousands of merozoites into the bloodstream, and hereby the merozoites invade the erythrocytes. The human malarial species mostly invades the Red blood cells. Plasmodium vivax has a very special affinity for Red blood cells (RBCs) that have developed from the bone marrow and are only 14 days old. The Plasmodium falciparum has lower affinity for young RBCs and thus, has significant ability to infect the older cells. The blood stages of malarial infection are responsible for most of the symptoms and pathologies, which are associated with malaria. This causes Plasmodium falciparum-infected erythrocytes to cytoadhere inside the small blood vessels of brain, kidneys, and other affected organs. There also occurs rosetting, which can be defined as the adherence of uninfected RBCs. Both of these processes, cytoadherence and rosetting, interfere with microcirculatory flow and metabolism of vital organs. The infected erythrocytes are host to the replicating malarial parasites for 48 hours before bursting, thus releasing the daughter merozoites. Parasitemia caused by Plasmodium vivax is symptomatically milder than is the one caused by $P$. falciparum. The merozoites thus produced after being released following rupture of the infected red cell get attached to the surface of the other uninfected red cells (Richa et al., 2017). 
Besides the above-described processes, the hallmark of falciparum malaria in pregnancy is the sequestration of parasites in the placenta. Sequestered parasites escape host defense mechanisms, such as splenic processing and filtration. Sequestration is not known to occur in the benign malarias due to $P$. vivax, $P$. ovale, and P. malariae (Richa et al., 2017).

\section{IRON DEFICIENCY AND PROTECTION FROM MALARIA}

Iron is found to be the major limiting factor for the growth of many protozoan or bacterial pathogens. Proliferation of Plasmodium requires iron, both during the pre-erythrocytic stage as well as the erythrocytic stage of infection. Malaria itself causes major disturbances in distribution and utilization of iron, through mechanisms, such as haemolysis, release of haeme, dyserythropoiesis, deposition of iron in macrophages, and reduced absorption of dietary iron. In vitro growth of malaria parasite could be, therefore, restricted by the use of iron-chelating agents, such as desferrioxamine (Spottiswoode et al., 2014). This has been found in murine models of malaria infection, and also in malaria-infected monkeys. Consequently, iron-chelating agents have been reviewed as an adjunct or primary antimalarial therapy. A better understanding of how Plasmodium parasites acquire iron during their various life stages might help in attaining success in the development of antimalarial drugs that are targeted toward the parasite's access to iron. Deficiency of iron in humans is likely to provide protection against severe malaria. On the contrary, supplementation with iron increases risks of infection and severity of disease (Spottiswoode $e t$ al., 2014). Concomitant deficiency of iron is usually treated after resolution of parasitemia.

\section{IRON REQUIREMENTS AND REGULATION DURING PREGNANCY}

During pregnancy, physiologic iron demands increase substantially to support fetoplacental development and maternal adaptation to pregnancy. Baseline maternal body iron losses during 9 month have been estimated at approximately $230 \mathrm{mg}$ (Allison and Elizabeta, 2017) and would be higher were it not for the cessation of menstruation. The development of the placenta and fetus requires approximately $360 \mathrm{mg} \mathrm{Fe}$. An additional $450 \mathrm{mg} \mathrm{Fe}$ is needed to expand maternal red blood cell (RBC) mass during pregnancy. Thus, approximately $1 \mathrm{~g}$ of iron must be acquired during pregnancy to preserve the maternal iron balance and support fetoplacental development. Some of that iron is recycled after pregnancy when the erythrocyte mass contracts to prepregnancy concentrations with the exception of the iron that is lost through bleeding at delivery (approximately $150 \mathrm{mg}$ ). Therefore, the average net pregnancy related loss of iron to the mother has been estimated to be $740 \mathrm{mg}$. However, iron requirements are not uniform throughout the 3 trimesters of pregnancy. In the first trimester, the requirements (estimated at approximately $0.8 \mathrm{mg} / \mathrm{d}$ ) are lower than before pregnancy because menstruation stops. As pregnancy advances, maternal RBC mass increases and placental and fetal growth accelerates, which result in the rise in physiologic iron requirements to $3.0-7.5 \mathrm{mg} / \mathrm{d}$ in the third trimester (Allison and Elizabeta, 2017).

To meet the accelerating physiologic iron requirements, both dietary iron absorption and the mobilization of iron from stores need to increase. Many women enter pregnancy with insufficient iron stores to meet the needs of the pregnancy. In the United States, the prevalence of iron deficiency (ID) in women of childbearing age has been reported to be $12 \%$ with a higher rate in Black and Hispanic women (19\% and 22\%, respectively). Because ID and ID anaemia (IDA) during pregnancy have been associated with adverse outcomes for the mother and the child, including increased risk of maternal mortality, premature birth, low birth weight, and neuro developmental impairment in infants (Allison and Elizabeta, 2017), iron supplementation has been nearly universally recommended during pregnancy. Nevertheless, in the developed world, more women are iron replete than iron deficient when they become pregnant, thus prompting considerations of potential risks of indiscriminate iron supplementation.

As assessed by the uptake of stable or radioactive iron isotopes, nonhaeme iron absorption during pregnancy increases as gestation progresses. It is likely that haeme absorption increases in a similar manner (Allison and Elizabeta, 2017). The regulation of iron availability during pregnancy is at least in part dependent on maternal hepcidin concentrations. Hepcidin, which is an ironregulatory hormone, is produced by the liver and controls plasma iron concentrations and tissue iron distribution. Hepcidin acts by inhibiting the following major iron flows into plasma: intestinal iron absorption, release from macrophages that recycle iron from old RBCs, and mobilization of stored iron from the liver. Hepcidin exerts its effects through its receptor 
the iron exporter ferroportin. Ferroportin is expressed in all the tissues that actively export iron into plasma. Hepcidin binds to ferroportin and triggers its degradation, resulting in iron sequestration in target cells and decreased iron flow into plasma. Thus, iron delivery to consuming tissues (e.g., bone marrow and placenta with fetus) is inversely correlated with hepcidin concentrations.

Hepcidin concentrations measured in either serum or urine were not correlated with inflammatory markers in healthy pregnancies, including those with multiple gestations ( $\mathrm{Ru}$ et al., 2016), thereby suggesting that the mild inflammation that occurs in healthy pregnancies is not sufficient to increase hepcidin. However, it is possible that hepcidin may be inappropriately increased in complicated pregnancies that are associated with more intense inflammation, and this increase could compromise iron availability during pregnancy. Elevated hepcidin would also be expected to impair iron absorption from supplements that are commonly prescribed to pregnant women and could even impair the efficacy of intravenous iron therapy by trapping iron in macrophages. Mildly elevated serum hepcidin concentrations have been reported in obese compared with lean pregnant women $(\mathrm{Ru}$ et al., 2016) and in pre-eclamptic compared with healthy pregnancies. These concentrations did not have an obvious negative impact on haematologic or iron variables in the mother or neonate in these studies, suggesting that hepcidin concentrations were still sufficiently low to allow effective iron utilization during pregnancy. Systematic studies of hepcidin in complicated pregnancies are needed to determine the extent of hepcidin elevation in different conditions and the impact of elevated hepcidin on pregnancy outcomes. Apart from the hepcidin-dependent mechanism that regulates iron absorption and recycling, additional hepcidinindependent mechanisms may exist in pregnancies, but this possibility remains to be characterized.

\section{Changes IN IRON STATUS DURING PREGNANCY AND IN MALARIA INFECTION}

The serum ferritin (SF) concentration is the most frequently used marker of iron stores. Ferritin is secreted mostly by macrophages and, to a lesser extent, by hepatocytes, in proportion to their intracellular iron contents; thus, SF is proportional to body iron stores. However, because ferritin production is also regulated by inflammatory cytokines, SF may not accurately reflect iron stores in the presence of inflammation. In pregnancy, SF concentrations gradually decrease to reach the lowest concentrations in the third trimester (Larsson et al., 2008).

In addition to haemodilution, this decrease likely reflects efficient iron mobilization from stores in agreement with the progressive hepcidin decrease during pregnancy. Iron supplementation results in a lesser SF decrease in the third trimester. Higher SF concentrations in the second or third trimester (Allison and Elizabeta, 2017) are associated with less favorable pregnancy outcomes including increased risk of preterm delivery. However, apart from reflecting higher iron stores in the mother, higher SF could also reflect the presence of inflammation in complicated pregnancies or the failure of the plasma volume to expand. Similar to other iron variables, serum iron and transferrin saturation (TSAT) both decrease during pregnancy but less so in iron-supplemented pregnancies (Allison and Elizabeta, 2017). The plasma iron compartment is very small compared with iron stores (several milligrams compared with several hundred milligrams), is subject to diurnal variation, and can change rapidly, e.g., after iron ingestion. Because of these effects, serum iron and transferrin saturation (TSAT) are inferior to SF in diagnosing ID. sTfR is generated by cleavage and by vesicular shedding of transferrin receptor 1 (TfR1) from the plasma membrane during erythroid maturation. The amount of soluble transferrin receptors (sTfR) reflects both the number of young erythrocytes and the degree of their iron deficiency (ID) because cellular TfR1 concentrations are regulated by intracellular iron via the iron responsive element-binding proteins (IRPs) IRP1 and IRP2. In pregnancy, sTfR concentrations do not seem to change compared with nonpregnant concentrations unless maternal erythropoiesis is iron deficient (Allison and Elizabeta, 2017). Thus, sTfR concentration may only mildly increase by the third trimester in the iron-replete population but increase substantially in women with IDA. Furthermore, because sTfR is not regulated by inflammation, STfR is a better indicator of iron-deficient erythropoiesis than SF is in the presence of inflammation.

(Susanne et al.,2011) also found no significant effect of peripheral or placental Plasmodium falciparum infection on cord-blood concentrations of hemoglobin, CRP, and sTfR and only a slightly effect on ferritin. Moreover, among Nigerian women, placental infection was strongly associated with maternal anemia but not with the mean hematocrit in their newborns. Blood concentrations are increased several folds 
in patients with iron deficiency, whereas they remain within the normal range in those with iron overload and anemia of chronic disease. Serum sTfR concentration is not affected by pregnancy unless the patient is also iron deficient (Ifeyinwa et al., 2017).The percent human transferrin in asymptomatic pregnant women with malaria infection is higher than was observed in the uninfected counterparts although no significant difference was observed. Although studies have reported that concentrations of serum iron and transferrin are low in the presence of acute phase reaction (Onyenekwe $e t$ al., 2010).

\section{LABORATORY SIGNIFICANCE AND DIAGNOSIS OF IRON}

The measurement of haematologic indicators in general and haemoglobin in particular is widely available in clinical and research laboratories by flow cytometry on fully automated cell counters. These instruments can count different blood cells with good precision and high sample throughput. Battery-operated, hand-held haemoglobinometers can provide on-the-spot results from a single drop of blood collected in the field. However, these instruments require freshly collected blood, and if capillary blood is used, the proper sampling technique is critical to obtaining a valid specimen (Christine and Anne, 2017). The measurement of serum-based biochemical indicators is generally carried out on fully-automated clinical analyzers available in many laboratories. Protein-based indicators such as SF, transferrin, and soluble transferrin sTfR are measured by immunoassays such as ELISA, whereas serum iron, TIBC, and UIBC are measured on chemistry and auto analyzers by using a colorimetric reaction with ferrine or ferrozine as a chromogen to form a color complex with iron. Most clinical analyzers measure UIBC because it is more easily automated than Total iron Biding Capacity (TIBC). The measurement precision of UIBC is good at high concentrations as found in iron depletion but worse at low concentrations in the presence of iron overload. Red cell counts, haemoglobin, mean corpuscular volume (MCV) hemoglobin concentration (MCHC), and red cell distribution width (RDW) can be used to determine the iron level (Salou et al., 2019).

\section{MANAGEMENT OF MALARIA AND IRON LEVEL IN PREGNANCY}

Malaria during pregnancy must be managed as an emergency situation, preferably by a multidisciplinary team comprising an intensive care specialist, infectious disease specialist, obstetrician, neonatologist, etc (RCOG, 2010). Women, who are recovering from an episode of malaria during pregnancy, are advised to have regular absolute neutrophil count, including assessment of maternal hemoglobin, platelet and glucose levels, and fetal growth scans. Treatment of choice for severe falciparum malaria is Intra-Venous (IV) artesunate. The IV quinine may be used, if artesunate is not available (RCOG, 2010). Blood films must be monitored at every 24 hourly intervals. However, the blood film must be repeated in case of clinical deterioration. Patient's hospitalization is important for ensuring compliance to therapy. The 7-day course of quinine may be associated with significant adverse effects, principally cinchonism, which may include signs and symptoms, such as tinnitus, headache, nausea, diarrhea, altered auditory acuity, and blurred vision. This may be frequently a cause of noncompliance, which may eventually result in the failure of therapy (RCOG, 2010).

Uncomplicated $P$. falciparum (or mixed infections, such as $P$. falciparum and $P$. vivax) can be treated with drugs, such as quinine and clindamycin. Chloroquine can be used for treating infection with $P$. vivax, $P$. ovale, or $P$. malariae. Primaquine should not be preferably used during pregnancy. The clinician should switch to IV therapy in cases of persistent vomiting. Antipyretics (paracetamol in standard doses) must be used for the treatment of fever. Prompt treatment of fever in malaria is necessary because it has been found to be associated with complications, such as premature labor and fetal distress. Paracetamol should be prescribed in the dosage of $1 \mathrm{gm}$ every 4 to 6 hours (to a maximum of $4 \mathrm{mg} /$ day). Women with malaria must be screened for anemia and treated appropriately (Richa et al., 2017). Women should be advised about the risk of recurrence of malaria, and a suitable followup plan must be devised. This may involve repeating the blood film in case the symptoms or fever returns. Infections, which recur following treatment, are likely to be intrinsically less sensitive to the drugs that have been previously used against them. The options for treatment of recurrent infection in pregnancy in the United Kingdom are limited. However, if quinine and clindamycin have failed as first-line treatment, an alternative should be considered. Atovaquone-proguanil artesunate and dihydroartemisinin-piperaquine have been 
effectively used in pregnant women with multiple recurrent infections (Richa et al., 2017). The WHO has recommended a 7-day regimen of artesunate in the dosage of 2 $\mathrm{mg} / \mathrm{kg} /$ day or $100 \mathrm{mg}$ daily for 7 days and clindamycin in the dosage $450 \mathrm{mg}$ three times daily for 7 days. The general rule of thumb is that the consequences of malaria are more harmful to the woman and her baby in comparison with the side effects related to the use of antimalarial medications.

\section{CONCLUSION}

Iron is found to be the major limiting factor for the growth of many protozoan or bacterial pathogens. Proliferation of Plasmodium requires iron, both during the pre-erythrocytic stage as well as the erythrocytic stage of infection. Malaria itself causes major disturbances in distribution and utilization of iron, through mechanisms, such as haemolysis, release of haeme, dyserythropoiesis, deposition of iron in macrophages, and reduced absorption of dietary iron. In vitro growth of malaria parasite could be, therefore, restricted by the use of ironchelating agents, such as desferrioxamine. Several hypotheses have been provided as to why malaria infection may be less prevalent among people with iron deficiency. Iron is vital for the survival of the malaria parasite and the parasite is unable to thrive in iron-deficient environment. Specifically, iron deficiency suppresses erythropoiesis, the production of red blood cells, thereby reducing the opportunity for parasite to infect the host.

\section{REFERENCES}

[1] Akinbo, F. O., Alabi, L. O., and Aiyeyemi, J. A. (2019) Micronutrient deficiencies among pregnant women with Plasmodium falciparum infection in Owo, Ondo State, Nigeria. Africa Journal of Clininical and Experimental Microbiology. 20 (2): 127 - 136.

[2] Andrew, M. P., Hala, G., Cono, D., and Sharon, E. C. (2010) Iron metabolism and malaria. Food and Nutrition Bulletin, 28 (4) S524-S536.

[3] Bao, W., Rong, Y., Rong, S., Liu L. (2012) Dietary iron intake, body iron stores, and the risk of type 2 diabetes: a systematic review and meta-analysis. Biomedical central Medicine. 10:119.

[4] Bothwell T. H. (2000) Iron requirements in pregnancy and strategies to meet them. Am J Clin Nutr 72: 257-264.

[5] Casgrain, A., Collings, R., Harvey, L.J., Hooper, L., and Fairweather-Tait, S.J., (2012) Effect of iron intake on iron status: a systematic review and meta-analysis of randomized controlled trials. American Journal of Clinical Nutrition; 96:768-780.

[6] Centers for Disease Control and Prevention. Recommendations to prevent and control iron deficiency in the United States. MMWR Recomm Rep. 1998; 47 (RR-3): 1-29.

[7] Chen, W., Dailey, H. A., Paw, P. H. (2010) Ferrochelatase forms an oligomeric complex with mitoferrin-1 and Abeb10 for erythroid heme biosynthesis. Blood 2010; 116: 628-630.

[8] Choi, J., Masaratana, P., Latunde-Dada, G.O., Arno, M., Simpson, R.J., and McKie A.T. (2012) .

[9] Duodenal reductase activity and spleen iron stores are reduced and erythropoiesis is abnormal in Dcytb knockout mice exposed to hypoxic conditions. Journal of Nutrition. 142:1929-1934.

[10] Dreyfuss M. L., Stoltful R. J., Shrestha J. B., Pradhan E.K., LeClerg S.C., (2000) Hookworms, malaria and Vitamin A deficiency contribute to anaemia and iron deficiency among pregnant women in the plains of Nepal. J of Nutr. 130: 2527-2536.

[11] Fuqua, B.K., Vulpe, C.D, and Anderson, G.J. (2012) Intestinal iron absorption. Journal of Trace Elements Medicine and Biology; 26:115119.

[12] Gayen, D., Sarkar, S.N., Datta, S.K., and Datta K. (2013) Comparative analysis of nutritional compositions of transgenic high iron rice with its non-transgenic counterpart. Food Chemistry. 138:835-840.

[13] Jones, E., Pasricha, S.R., Allen, A., Evans, P., Fisher, C.A., Wray, K., Premawardhena, A., Bandara, D., Perera, A., and Webster C. (2015) Hepcidin is suppressed by erythropoiesis in hemoglobin $\mathrm{E}$ beta-thalassemia and betathalassemia trait. Blood 125:873-880.

[14] Koenig, M.D., Tussing-Humphreys, L., Day, J., Cadwell, B., and Nemeth, E. (2014) Hepcidin and iron homeostasis during pregnancy. Nutrients. 6:3062-3083.

[15] Larsson, A., Palm, M., Hansson, L. O., and Axelsson O. (2008). Reference values for clinical chemistry tests during normal pregnancy. BJOG; 115:874-878.

[16] Natasha, S., Patrick, E. D., and Hal, D. (2014) Iron, anemia and hepcidin in malaria. Frontiers in pharmacology. 5(121)1-10.

[17] Okamgba, O.C., Nwosu, D.C., Nwobodo, E.I., Agu, G.C., Ozims, S.J., Obeagu, E.I., Ibanga, I.E., Obioma- Elemba, I.E., Ihekaire, D.E., Obasi, C.C., Amah, H.C., Ekendu, M.I., and Ifeanyichukwu, M.O. (2017) Iron Status of Pregnant and Post-Partum Women with Malaria Parasitaemia in Aba Abia State, Nigeria. Annals of Clinical and Laboratory Research. 5:4:206 
[18] Paul, V. K., Sachdev, H. S., and Mavalankar, D. (2011) Reproductive health, and child health and nutrition in India: meeting the challenge. Lancet. 377(9762): 332349.

[19] Royal College of Obstetricians and Gynaecologists. (2010) Preventing malaria in pregnancy. (Green-top Guideline No. 54A).

[20] Ru, Y., Pressman, E., Cooper, E., Guillet, R., Katzman, P., Kent, T., Bacak, S., and O'Brien, K., (2016) Iron deficiency and anemia are prevalent in women with multiple gestations. American Journal of Clinical Nutrition; 104: $1052-60$

[21] Spottiswoode, N., Duffy, P.E., and Drakesmith, H. (2014) Iron, anemia and hepcidin in malaria. Frontiers Pharmacology 5:125.

[22] Suragani, R.N., Zachariah, R.S., Velazquez, J.G., Liu, S., Sun, C.W., Townes, T.M., and Chen, J.J. (2012) Heme-regulated eif2alpha kinase activated Atf4 signaling pathway in oxidative stress and erythropoiesis. Blood. 119:5276-5284.

[23] White P. J., Broadley M. R: Biofortification of crops with seven mineral elements often lacking in human diets- iron, zinc, copper, calcium, magnesium, selenium and iodine. New Phytol 2009; 182: 49-84.

[24] WHO (2015). The global prevalence of anaemia in 2011. Geneva (Switzerland): WHO Document Production Services:1-48.

[25] Worwood M, May A., (2012) IDA and iron overload: In Vacie and Lewis Practical Haematology. (11 ${ }^{\text {th }}$ edn) Barbara J. B., Imelda B., Mike A. I., Lewis S. M., (eds) Elsevier ChurchHill Livingstone LTD. UK. pp. 175-200.

[26] Yersin, S., Favrat, B., Bodenmann, P., Cheseaux, M. (2012) Aneamia secondary to geophagia in a rich country? A case report (in French). Revue Medicale Suisse; 8:604-606.

Citation: Obeagu Emmanuel Ifeanyi, "Iron Status of Malarial Infected Pregnant Women: A Review", International Journal of Research Studies in Medical and Health Sciences. 2020; 5(1): 08-18.

Copyright: (C) 2020 Obeagu Emmanuel Ifeanyi, This is an open-access article distributed under the terms of the Creative Commons Attribution License, which permits unrestricted use, distribution, and reproduction in any medium, provided the original author and source are credited. 